\title{
Papua New Guinea Under the COVID-19 Pandemic and Public Health Support from the World Health Organization
}

\author{
Wenwen Lei ${ }^{1, \hbar}$
}

On March 20, 2020, the first positive case of coronavirus disease 2019 (COVID-19) was detected in Papua New Guinea (PNG). That same day, a state of emergency was declared in the country, thereby halting all incoming and domestic flights and limiting travel between provinces. Meanwhile, the PNG government established the National Control Centre (NCC) to specifically respond to the pandemic. The pandemic's second wave struck local areas in February 2021, with a corresponding surge in the number of positive cases (Figure 1A). As of March 15, 2021, 54,410 nasal samples had been collected and tested nationwide, with 2,351 confirmed positive cases and 26 deaths.

While these numbers are still lower than that in some other countries, there has been a sharp increase in cases and the World Health Organization (WHO) is highly concerned about the pandemic's potential to spread much wider (1). Due to low testing capacity and a shortage of testing personnel and reagents, the actual number of infections in PNG may be far higher than the reported number (2). The pandemic in PNG is suspected to be underestimated and has spread rapidly nationwide (Figure 1B). The number of cases in the capital city of Port Moresby has accounted for $47 \%$ of the country's total. Port Moresby General Hospital, the country's largest hospital, was overwhelmed at that time (2). In terms of health security threats, PNG is one of the most at-risk countries in the world (3). Dengue, tuberculosis, malaria, HIV, and diarrhea are present and ongoing threats for PNG (2). PNG also has a relatively weak healthcare system (3) and one of the world's lowest ratios of doctors; the World Bank figures for 2018 show the country had only 0.07 physicians per 1,000 people. This is well below the 2017 average for Pacific Small Island Developing States (0.5), the 2017 world average (1.6), and the 2017 level in the United States (2.6) (4). As of April 2, 2021, the country had 500 doctors, fewer than 4,000 nurses, fewer than 3,000 community health workers, and roughly 5,000 hospital beds for 9 million people (4).

From the early stages of the COVID-19 pandemic in 2020, the WHO deployed experts to the PNG government to provide technical support for case management, epidemiology, infection prevention and control, laboratory support, and information management (1). The $\mathrm{WHO}$ and related personnel worked in the NCC's Incident Management Team (IMT) framework under the leadership of the PNG National Department of Health (NDoH) staff. Global Outbreak Alert and Response Network (GOARN) experts were located within the IMT pillar areas, assisting in daily work, providing technical assistance, and supporting special projects. From November 4, 2020 to March 30, 2021, the WHO invited me, as a laboratory technical expert in response to the COVID-19 pandemic, to provide technical assistance for PNG. I am the first person successfully dispatched by GOARN from China during the COVID-19 pandemic, and the first Chinese expert to be deployed by an international organization to PNG for assistance in this pandemic.

During nearly 5 months of work, I engaged in communication with and coordinated with various organizations and departments such as the WHO Headquarters, WHO Western Pacific Region, local governments and the $\mathrm{NDoH}$, and provincial health authorities, and I worked in accordance with the PNG Response Plan for COVID-19. My colleagues and I have successfully held a series of national workshops on enhancing the diagnosis of and response to COVID-19 and aimed to improve PNG laboratories' diagnosis and detection capabilities. During January 20-23, 2021, I traveled to East New Britain Province to conduct theoretical and practical training in Nonga General Hospital for rapid detection of severe acute respiratory syndrome coronavirus 2 (SARS-CoV-2) antigens. The methods taught were later implemented nationwide. My colleagues and I have also evaluated and certified the laboratories in PNG with nucleic acid detection capacity. We have reviewed and assessed the COVID19 real-time reverse transcription polymerase chain reaction (rRT-PCR) assay and instrument platform, and laboratory design and space, for three private 
laboratories in the capital - Aspen Medical Laboratory, Sky Health \& Medical Services COVID19 Laboratory, and Pacific International Hospital. We issued an evaluation report providing suggestions on the diagnostic algorithm, test verification, and reporting process regarding COVID-19. The WHO Regional Director for the Western Pacific, upon learning of my work in PNG, expressed great pleasure and strong affirmation that WHO experts were supporting pandemic prevention and control in PNG.

I already engaged in COVID-19 pandemic control in Wuhan, Suifenhe, and Shulan cities and the Beijing Xinfadi Wholesale Market in China in 2020. The experience acquired there provided me with a vital foundation for my work in PNG. I believe communication, sharing, and learning are pivotal for controlling infectious diseases at the global scale.

As of September 28 2021, a total of 191,925 vaccine doses had been administered in PNG. The situation in PNG is excellent proof of the vital importance of vaccine fairness. Vaccines have contained the COVID19 pandemic for a long time, but with the increase in

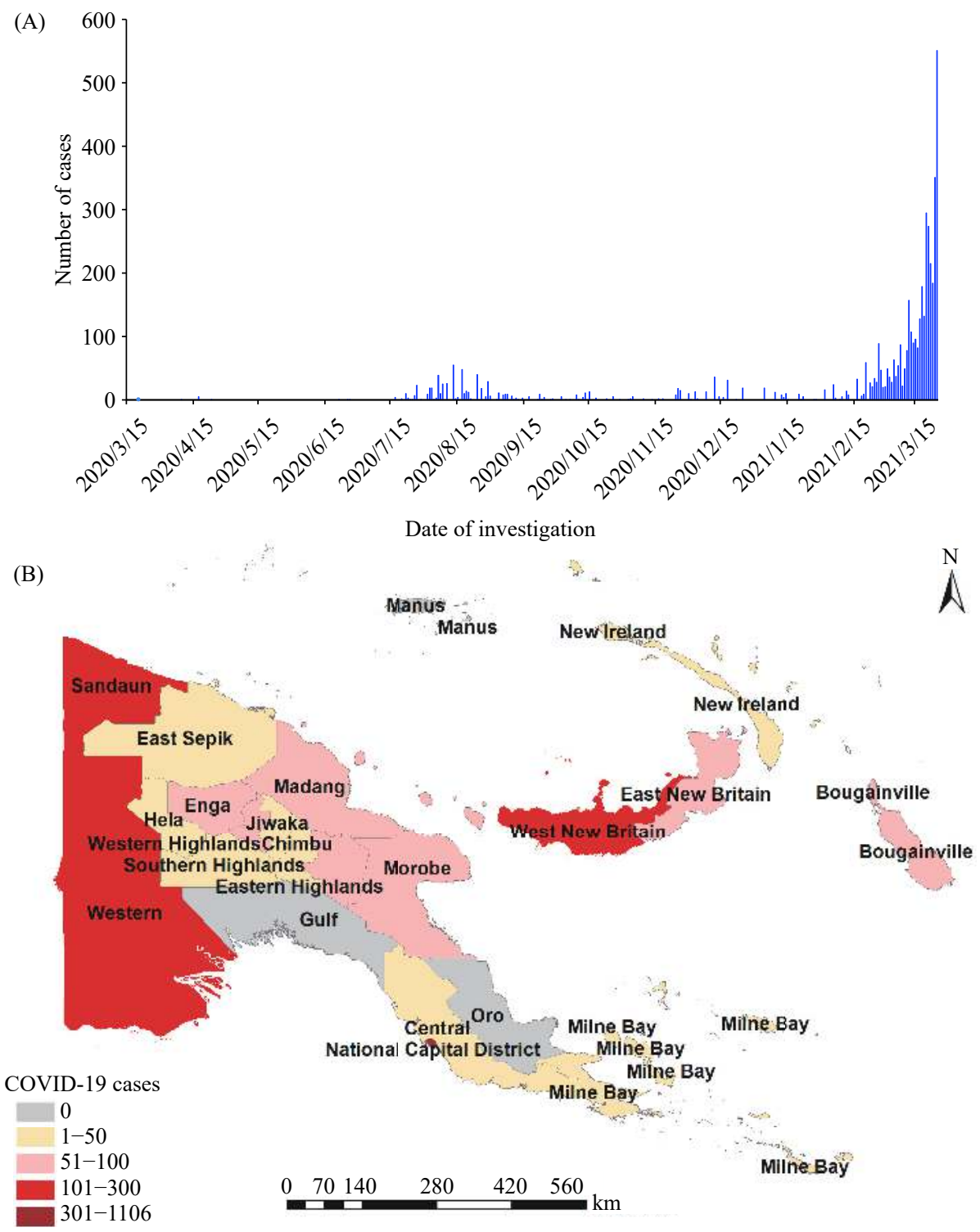

FIGURE 1. Coronavirus disease 2019 (COVID-19) pandemic in Papua New Guinea. (A) Papua New Guinea confirmed cases from March 15, 2020, to March 15, 2021. (B) Distribution of COVID-19 cases in Papua New Guinea as of March 15, 2021.

Note: The map was created using ArcGIS (version 10.4, Esri, Redlands, CA, USA). Data were collected from WHO website: https:// covid19.who.int/region/wpro/country/pg. 
the number of infections, fatigue from social restrictions, low levels of people's immunity, and fragile health systems, it is crucial to obtain more vaccines as soon as possible. In the future, vaccinations should be vigorously promoted in PNG, laboratory testing and monitoring capabilities strengthened at the same time - i.e., to strengthen the whole genome sequencing and RT-PCR infrastructure in the national public health plan-early detection and monitoring of new SARS-CoV-2 mutant strains strengthened, and detection capabilities expanded. These measures will play a greater role in controlling the epidemic.

Since the COVID-19 outbreak was declared a Public Health Emergency of International Concern, the WHO, through GOARN, has deployed more than 130 experts to 26 countries to provide support in enhancing laboratory capacity, epidemiology, infection prevention and control, case management, and data management (5). All these experts have greatly contributed to controlling the COVID-19 pandemic. Meanwhile, public health experts from China have been to countries including Iran, Iraq, Russia, and Italy to support local COVID-19 control and technique training, just as the supportive work in Western Africa during the Ebola epidemic (6-7). China is collaborating with different countries on Phase III clinical trials of the vaccines, and Chinese vaccines against COVID-19 have been shared with more than 100 countries and international organizations. Working together with experts from different countries under the WHO's coordination, China's public health experts are increasingly participating in global public health efforts to improve governance in this area and build a community concerned with the health of greater mankind.

Acknowledgments: Dr. Dapeng Luo, Dr. Anna Maalsen, Dr. George F. Gao, Dr. Guizhen Wu, Dr. William J. Liu, Dr. Xianyuan Du, and Dr. Idrissa Laybohr Kamara.

Conflicts of interest: No conflicts of interest.

doi: $10.46234 / \mathrm{ccdcw} 2021.214$

\# Corresponding author: Wenwen Lei, leiww@ivdc.chinacdc.cn.

${ }^{1}$ National Institute for Viral Disease Control and Prevention, Chinese Center for Disease Control and Prevention, Beijing, China.

Submitted: August 16, 2021; Accepted: September 08, 2021

\section{REFERENCES}

1. WHO. Director-General's opening remarks at the media briefing on COVID-19 - 16 April 2021. 2021. https://www.who.int/directorgeneral/speeches/detail/director-general-s-opening-remarks-at-the-mediabriefing-on-covid-19-16-april-2021. [2021-7-15].

2. Pryke J. Australia must take immediate action to stave off catastrophe in our nearest neighbour. The Australian. 2021. https://www.lowyinstitute. $\mathrm{org} /$ publications/australia-must-take-immediate-action-stave-catastropheour-nearest-neighbour\#. [2021-7-15].

3. Papua New Guinea Department of Health. Papua New Guinea emergency preparedness and response plan coronavirus diseases 2019 (COVID-19). Version 17, 2020. http://health.gov.pg/covid19/ PNGEPRPCOVID19.pdf. [2021-7-16].

4. Hollingsworth J. This country only has about 500 doctors for 9 million people. Now it's dealing with a COVID outbreak. CNN. 2021. https:// edition.cnn.com/2021/03/27/asia/papua-new-guinea-covid-outbreakintl-hnk-dst/index.html. [2021-7-15].

5. World Health Organization. COVID-19 response. Report by the director-general. EB148. 2021. https://apps.who.int/gb/ebwha/ pdf_files/EB148/B148_16-en.pdf. [2021-7-15].

6. Gao GF, Feng Y. On the ground in Sierra Leone. Science 2014;346(6209):666. http://dx.doi.org/10.1126/science.346.6209.666.

7. Liu WJ. On the ground in Western Africa: from the outbreak to the elapse of Ebola. Protein Cell 2016;7(9):621 - 3. http://dx.doi.org/10. 1007/s13238-016-0305-2. 